\title{
Radiotherapy Induced Middle Ear Morbidities in Head and Neck Cancer Patients
}

https://doi.org/10.47210/bjohns.2020.v28i3.322

Adity Chakraborty, ${ }^{1}$ Abhinandan Bhattacharjee, ${ }^{2}$ Arnab Purkaystha, ${ }^{3}$ Aakanksha Rathor, ${ }^{4}$ Ridip Mazumdar ${ }^{2}$

Introduction

ABSTRACT

Although post radiotherapy (RT) otological complications are complex and common, there is lack of data on its severity and spectrum. We intend to assess the incidence and severity of such ototoxicities in head neck cancer (HNCA) patients. Materials and Methods

One year prospective study was conducted on thirty cases of HNCA receiving radiotherapy. Audiometry and tympanometry changes were noted and severity is assessed using Common Toxicity Criteria (CTC) -v2.0 of the National Cancer Institute (NCI). Fisher's Exact Test with two-sided p value was used for comparison of the tympanometric changes.

$\underline{\text { Results }}$

The mean age was 55.4 years. The commonest site of HNCA was oral cavity (30\%) with $46.6 \%$ in stage II. $40 \%$ of cases were in grade II, followed by $27 \%$ cases in Grade III. The commonest complaint was pain (86.6\%), hearing loss (80\%) and 40\% post-RT cases reporting Type B curve (relative risk 0.67).

Conclusion

Post-irradiation pain and OME are a major concern in HNCA patients having grade II (NCI-CTC). Early detection and prophylactic measures will improve the quality of life in such group of patients. This result validates the need for formulating otological diagnostic strategy \& therapeutic measures in patients receiving RT to mitigate post-irradiation ototoxicities.

$\underline{\text { Keywords }}$

Head and Neck Neoplasms; Radiotherapy; Morbidity, Ear; Prospective Study

$\mathrm{H}$ ead and Neck Squamous Cell Carcinoma (HNSCC) has a high incidence of $54.48 \%$ in North-Eastern India ${ }^{1}$ and radiotherapy (RT) is a common modality of treatment. As such, RT induced toxicities like neurological complications and hearing impairment gain particular importance. These toxicities are primarily due to the complex anatomy and unavoidable exposure of non-target organs to irradiation in head and neck region. ${ }^{2}$

Otological morbidities pertaining to the external ear include reactions involving the preauricular region, the auricle, and the external auditory canal $(\mathrm{EAC})^{3,4}$ Other complications include eustachian tube dysfunction, OME, transient conductive hearing loss, tympanosclerosis, perforation, middle ear fibrosis and/ or ossicular atrophy. Morbidities involving the inner ear include tinnitus, labyrinthitis, canal paresis and vertigo/ balance problems. ${ }^{7}$ Several studies have also reported RT induced damage to the cochlear nerve leading to sensorineural hearing loss (SNHL). ${ }^{4,5,7,8}$

Despite the diversity, complexity and extent of the functional consequences, post-RT ototoxicity is sparsely

1 - Department of Oncology, Guwahati Medical College, Assam

2 - Department of ENT, Silchar Medical College, Assam 3 - Student, Silchar Medical College, Assam

4 - Department of Otorhinology, Head and Neck Surgery,

Stanford University School of Medicine, CA, USA

Corresponding author:

Dr Abhinandan Bhattacharjee

email: dr_abhinandan1@rediffmail.com 
reported in the radiation oncology literature. ${ }^{5}$ There is also lack of clear-cut data on incidence, type, severity and evaluation criteria of radiation induced ear toxicity ${ }^{2}$ in the Common Toxicity Criteria (CTC)-v2.0 of the National Cancer Institute. ${ }^{2}$ This is partly due to differences in irradiation schemes, dosage, fractionation techniques and lack of awareness of such morbidities. Moreover, in absence of any study on this subject from this region of India, we intend to evaluate the institutional incidence, spectrum and severity of RT induced ototoxicities.

\section{Materials and Methods}

Thirty cases of head and neck carcinoma (HNCA), treated with RT were taken up for the study. Informed consent was taken before the study. Patients with primary tumour of the auditory system or suffering from any auditory problem before initiation of radiation were not considered for this study. Patients with the history of incomplete radiotherapy treatment or not available for follow-up for more than 2 months were also excluded from the study.

Study subjects were examined before the start of radiotherapy (pre-RT), at 11th fraction of radiotherapy (2nd week RT) and after completion of radiotherapy (post-RT). Detailed history-taking, otoscopic examination and tympanometry were carried out. The CTC-v 2.0 of National Cancer Institute was used for toxicity grading. All data were recorded in the proforma and Fisher's Exact Test with two-sided p value was used to compare the tympanometric changes of middle ear in RT exposed \& non exposed ears. Ethics clearance for the study was obtained before starting the study.

\section{Results}

The effects of radiation were studied on 30 patients (60 ears) which included 30 ipsilateral ears and 30 contralateral ears. The mean age of study population and control subjects were 55.4 years and 46.13 years respectively. The commonest site of HNCA was the oral cavity $(30 \%) .46 .6 \%$ of patients presented at stage II and $36.6 \%$ at stage III \& above disease. (Table I)
Table I: Demographic characteristics of study population

\begin{tabular}{|c|c|c|}
\hline & $\begin{array}{c}\text { PATIENTS } \\
\text { UNDER } \\
\text { RT }(\mathbf{N}=\mathbf{3 0})\end{array}$ & $\begin{array}{c}\text { CONTROL } \\
(\mathbf{N}=\mathbf{3 0})\end{array}$ \\
\hline Age (Mean) & 55.4 & 46.13 \\
\hline \multicolumn{3}{|l|}{ Sex } \\
\hline Male & $22(73 \%)$ & \\
\hline Female & $8(27 \%)$ & \\
\hline Ipsilateral ears & 30 & \\
\hline Right & 18 & \\
\hline Left & 12 & \\
\hline Contralateral ears & 30 & \\
\hline Right & 12 & \\
\hline Left & 18 & \\
\hline \multicolumn{3}{|l|}{ Site of cancer } \\
\hline Oral Cavity & 9 & \\
\hline Oropharynx & 7 & \\
\hline Larynx/pharynx & 8 & \\
\hline Esophagus & 5 & \\
\hline \multicolumn{3}{|l|}{ Stage of disease } \\
\hline Stage I & 5 & \\
\hline Stage II & 14 & \\
\hline Stage III \& IV & 11 & \\
\hline
\end{tabular}

Radiation toxicity assessment grade: Using NCICTC, we clinically evaluated the irradiated cases and found $40 \%$ of cases in grade II, followed by $27 \%$ cases in Grade III. (Fig. 1)

Radiation induced ear morbidities: The commonest ear morbidity noted was pain, seen in 26 patients $(86.6 \%)$, followed by hearing loss in 24 patients $(80 \%)$. Radiation dermatitis, OME and ulceration of external ear were other common presentations. (Fig. 2)

Tympanometric evaluation of middle ear: 


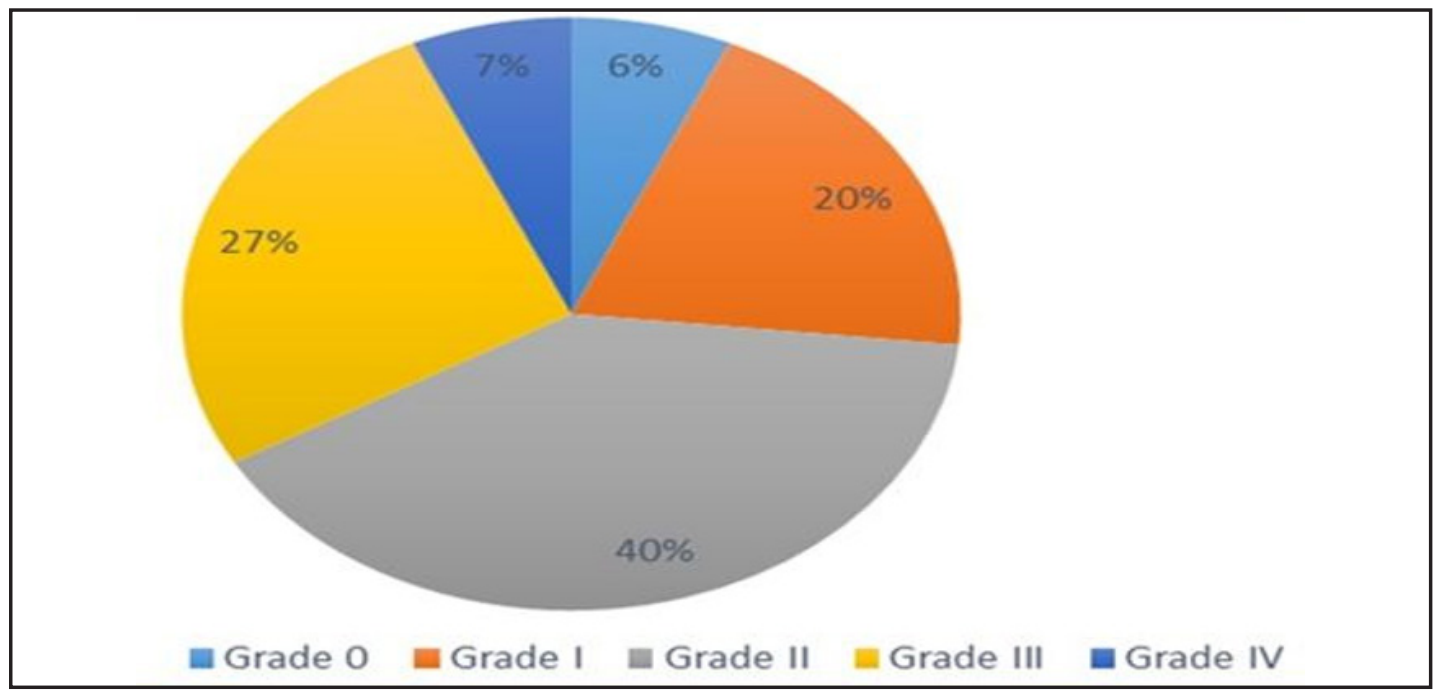

Fig.1. Radiation toxicity assessment grade (NCI-CTC) in study population

Tympanometry curves Type B \& Type C were seen in $40 \%$ and $20 \%$ cases respectively in RT-exposed ears. Fisher's Exact Test showed $\mathrm{p}$ value of 0.196 which was not significant. The Relative Risk was calculated to be 0.6667 and $95 \%$ Confidence Interval of 0.3936 to 1.129 (Table II)

\section{Discussion}

RT is one of the mainstays of treatment for HNCA. Whole or parts of the auditory system receive high doses of RT thereby causing various RT-induced injuries to the external, middle and inner ear. ${ }^{3}$ We assessed the effect of radiation on the external ear and middle ear in 30 (thirty) HNCA patients. These cases received RT with once-daily fractionation with the fraction size of $200 \mathrm{cGy}$ receiving a total radiation dose of 65-70 Gy at

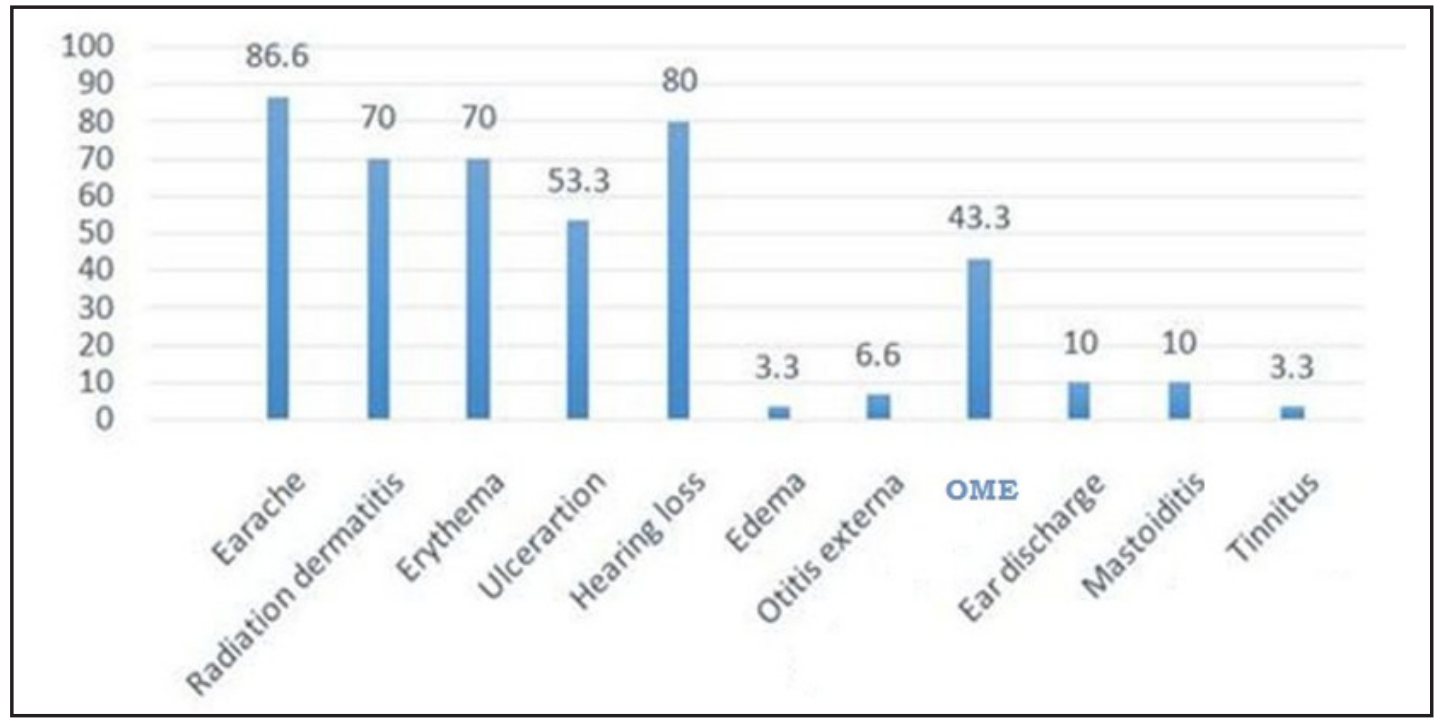

Fig.2. Spectrum of post RT condition of the ears 
Table II : Tympanometry results in patients undergoing RT.

\begin{tabular}{|c|c|c|c|c|}
\hline TYPE OF CURVE & A & B & C & P VALUE(<.05) \\
\hline Ipsilateral & & & & \\
\hline Pre-RT & 18 & 8 & 4 & $0.1213(\mathrm{~ns})$ \\
\hline Post-RT & 12 & 12 & 6 & \multirow{2}{*}{$0.7075(\mathrm{~ns})$} \\
\hline Contralateral & 18 & 8 & 4 & \multirow{2}{*}{3} \\
\hline Pre-RT & 16 & 10 & & \\
\hline
\end{tabular}

completion.

Although there are a number of toxicity assessment methods reported in literature, we used the National Cancer Institute Common Toxicity Criteria Grading system (NCI-CTC). Scoring systems like Radiation Therapy Oncology Group (RTOG) criteria and Late Effects of Normal Tissue/Somatic Objective Management Analytic (LENT/SOMA) scoring system are generally suited for late effects which were not assessed in our study. There are also several limitations in this system like lack of distinction between external, middle and inner ear toxicity and too narrow hearing loss category. ${ }^{2}$

The CTC grading has been reported in chemotherapy studies and its use in radiation toxicities is a new application that was taken up in our study. CTC Grade II was the commonest in our study (40\%) followed by Grade III (26.6\%) (Fig 1). This points to the fact that more than half of the irradiated cases had some form of toxicity like radiation dermatitis, earache, middle ear effusion or hearing loss. We observed that clinical assessment of radiation toxicities using the CTC Grade was beneficial in the comprehensive work-up of the patients and helped to categorise the severity of the different ear toxicities. This system was also practically easy for use by allied health workers involved in cancer care and can be easily interpreted.

External ear morbidities include reactions involving the preauricular region, the auricle, and the external auditory canal (EAC). ${ }^{3,4}$ In our study, about $86.6 \%$ cases suffered from pain in and around the ear followed by hearing loss $(80 \%)$, which may be conductive $(70 \%)$ or sensorineural (30\%) (Fig 2). Acute reactions commonly encountered during RT included otitis externa, erythema, dry and moist desquamation, and ulceration of the skin of the pinna and the EAC resulting in mild to severe pain and otorrhea. Radiation may induce osteitis and vasculitis of the surrounding soft tissue of the EAC, deep ulceration of the EAC, and osteonecrosis leading to refractory otitis externa. Damage from osteoradionecrosis to nearby structures may be due to the patients' predisposition to aggressive or chronic infectious processes or tissue destruction by the necrosis. ${ }^{9}$

Pre-existing otitis externa or localised but progressive infections may result from bone necrosis with persistent suppuration. Some studies reported life-threatening complications such as multiple brain abscesses, internal carotid artery aneurysm, aggressive EAC cholesteatoma, sigmoid sinus thrombosis, and otitic meningitis associated with temporal bone osteonecrosis. ${ }^{10}$ The incidence of mastoiditis in our study was $10 \%$. However, tinnitus was seen in only $3.3 \%$ cases as compared to $16.2 \%$ in other studies5. Although morbidities associated with the inner ear also include a wide variety of manifestations such as labyrinthitis, canal paresis and vertigo/balance problems, we did not encounter any such toxicities in our study subjects. Another study demonstrated statistically significant difference in incidence of toxicity $(67 \%, p=.0085)$ in patients receiving cochlear dose of greater than or equal to $60 \mathrm{~Gy}$, in comparison to less than $60 \mathrm{~Gy}$ (Table I). ${ }^{11}$ 
In the middle ear, eustachian tube dysfunction and otitis media with effusion (OME) remained the most common complications (43.3\%). Type B tympanogram was obtained in $40 \%$ of Post-RT cases and Type C curve was seen in $26.6 \%$ cases. However, these findings were not statistically significant (Table 2). Some studies reported that Type B curve can be a prognostic factor for significant sensorineural hearing loss (SNHL) than those with type A tympanograms ( $88 \%$ versus $15 \%$, p $=.02) .{ }^{11}$ Thickening of the tympanic membrane (TM) with sclerosis and perforation in addition to middle ear fibrosis and/or ossicular atrophy has also been reported in higher doses of RT. ${ }^{11}$ We found active mucosal otitis media in $10 \%$ cases which is due to TM perforation and persistent otorrhea. This follows fibrovascular granulation tissue proliferation, sometimes with inflammatory polyp formation6. RT induced changes in the mucosa of mesotympanum shows marked changes in the epithelium, connective tissue and endothelial cells of blood capillaries. Some studies reported that the prevalence of middle ear complications decreases at 10 years after RT due to resolved inflammatory reaction and improved Eustachian tube function. ${ }^{12}$

\section{Conclusion}

Despite the functional consequences, radiation induced otological injuries remain under-evaluated and underreported. Our study validates the need for formulating otological diagnostic strategy \& therapeutic measures in patients receiving RT to mitigate post-irradiation ototoxicities. We suggest routine evaluation of the auditory system during radiation therapy as early detection \& prophylactic measures will improve the quality of life in such group of patients. As post RT ear morbidities were noted in a good majority of HNCA patients, adopting CTC grades in the evaluation for ototoxicity alongwith tympanometry assessment should be routinely considered in the follow-up of HNCA cases receiving radiotherapy.

\section{References}

1. Bhattacharjee A, Chakraborty A, Purkaystha P. Prevalence of Head and Neck Cancer in the North - East India - An institutional study. Indian Journal Otolaryngol Head Neck Surg. 2006; $58: 1 ; 15-9$

2. Barbara A, Jereckzek Fossa, Andrzej Zarowski et al Radiotherapy induced ear toxicity. Cancer treatment reviews 2003; 29:417-30

3. Borsanyi SJ, Blanchard CL. Ionizing radiation and the ear. JAMA 1962; 181:958-61

4. Carls JL, Mendenhall WM, Morris CG, et al. External auditory canal stenosis after radiation therapy. Laryngoscope 2002; $112: 1975-8$

5. Bhandare N, Antonelli PJ, Morris CG, Malayapa RS, Mendenhall WM. Ototoxicity after radiotherapy for head and neck tumors. International Journal of Radiation Oncology Biology Physics 2007; 67(2): 469-79

6. Jereczek-Fossa BA, Zarowski A, Milani F, Orecchia R. Radiotherapy-induced ear toxicity. Cancer Treat Rev. 2003; 29(5):417-30. doi: 10.1016/s0305-7372(03)00066-5

7. Bhide SA, Harrington KJ, Nutting CM. Otological toxicity after postoperative radiotherapy for parotid tumours. Clinical Oncology 2007; 19:77-82

8. Raaijmakers E, Engelen AM. Is sensorineural hearing loss a possible side effect of nasopharyngeal and parotid irradiation? A systematic review of the literature. Radiother Oncol. 2002; 65(1):1-7. doi: 10.1016/s0167-8140(02)00211-6

9. Sikand A, Longridge N. CSF otorrhea complicating osteoradionecrosis of the temporal bone. J Otolaryngol. 1991; 20:209-11

10. Leonetti JP, Origitano $\mathrm{T}$, Anderson $\mathrm{D}$, et al. Intracranial complications of temporal bone osteoradionecrosis. Am J Otol.1997; 18:223-8

11. Chen WC, Liao CT, Tsai HC, Yeh JY, Wang CC, Tang SG, Hong JH. Radiation-induced hearing impairment in patients treated for malignant parotid tumor. Ann Otol Rhinol Laryngol. 1999; 108(12):1159-64

12. Young YH, Cheng PW, Ko JY. A 10-year longitudinal study of tubal function in patients with nasopharyngeal carcinoma after irradiation. Arch Otolaryngol Head Neck Surg. 1997; 123:9458 . 